\title{
Metallicity Mapping with gri Photometry: The Virgo Overdensity and the Halos of the Galaxy
}

\author{
Timothy C. Beers ${ }^{1,2}$, Deokkeun An ${ }^{3}$, Jennifer A. Johnson ${ }^{4}$, Marc H. \\ Pinsonneault ${ }^{4}$, Donald M. Terndrup ${ }^{4}$, Franck Delahaye ${ }^{5}$, Young Sun \\ Lee $^{1,2}$, Thomas Masseron ${ }^{4}$, Daniela Carollo ${ }^{6,7}$ \& Brian Yanny ${ }^{8}$ \\ ${ }^{1}$ Department of Physics \& Astronomy, Michigan State University, \\ email: beers@pa.msu.edu \\ ${ }^{2}$ Joint Institute for Nuclear Astrophysics \\ ${ }^{3}$ IPAC, Caltech \\ ${ }^{4}$ Department of Astronomy, Ohio State University \\ ${ }^{5}$ CEA-Saclay, DSM/IRFU/Service dAstrophysique, France \\ ${ }^{6}$ Research School of Astronomy \& Astrophysics, ANU, Australia \\ ${ }^{7}$ INAF, Osservatorio Astronomico di Torino, \\ ${ }^{8}$ FermiLab
}

\begin{abstract}
We describe the methodology required for estimation of photometric estimates of metallicity based on the SDSS gri passbands, which can be used to probe the properties of mainsequence stars beyond $\sim 10 \mathrm{kpc}$, complementing studies of nearby stars from more metallicitysensitive color indices that involve the $u$ passband. As a first application of this approach, we determine photometric metal abundance estimates for individual main-sequence stars in the Virgo Overdensity, which covers almost $1000 \mathrm{deg}^{2}$ on the sky, based on a calibration of the metallicity sensitivity of stellar isochrones in the gri filter passbands using field stars with well-determined spectroscopic metal abundances. Despite the low precision of the method for individual stars, internal errors of $\sigma_{[\mathrm{Fe} / \mathrm{H}]} \sim 0.1$ dex can be achieved for bulk stellar populations. The global metal abundance of the Virgo Overdensity determined in this way is $\langle[\mathrm{Fe} / \mathrm{H}]\rangle=$ $-2.0 \pm 0.1$ (internal) \pm 0.5 (systematic), from photometric measurements of 0.7 million stars with heliocentric distances from $\sim 10 \mathrm{kpc}$ to $\sim 20 \mathrm{kpc}$. A preliminary metallicity map, based on results for 2.9 million stars in the northern SDSS DR-7 footprint, exhibits a shift to lower metallicities as one proceeds from the inner- to the outer-halo population, consistent with recent interpretation of the kinematics of local samples of stars with spectroscopically available metallicity estimates and full space motions.
\end{abstract}

Keywords. astronomical data bases: surveys, Galaxy: halo, structure, methods: data analysis, stars: abundances

\section{Introduction}

The structure, chemistry, and kinematics of the stellar halo of the Galaxy, with its predominantly old and metal-poor populations, collectively preserve a detailed record of our Galaxy's formation in the early universe. Thanks to large-area surveys such as the Sloan Digital Sky Survey (SDSS; York et al. 2000), recent studies have revealed that the halo is marked by numerous stellar substructures. The presence of these lumpy and complex substructures (both in real space and in phase space) are in qualitative agreement with models for the formation of the stellar halo through the hierarchical merging and accretion of low-mass sub-halos (e.g., Bullock \& Johnston 2005). Among 
the various substructures discovered to date, the Virgo Overdensity (VOD) is one of the most striking. It was discovered as a stellar overdensity of main-sequence stars in SDSS; star counts in the region toward Virgo are enhanced by a factor of two above the background stellar distribution (Jurić et al. 2008). The overdensity seems to be associated with clumps of RR Lyrae stars and turn-off stars, but it is less likely to be connected with the leading tidal tail of the Sagittarius dwarf galaxy (e.g., Newberg et al. 2007, and references therein). At present, metallicity estimation from broadband photometry is the only practical means of obtaining metal abundances for a large number of faint objects such as those in the VOD. Such methods are based on the relative sensitivity of stellar colors to photospheric abundances over a wide wavelength baseline. The clear advantage of using a photometric metallicity technique is the efficiency of estimating metallicities for individual main-sequence stars, which are the most plentiful and representative sample of stellar populations.

Ivezić et al. (2008a) constructed photometric metallicity relations in the $u-g$ vs. $g-r$ plane using SDSS filter passbands, and studied the abundance structures of the Galaxy with an accuracy of $\sim 0.2$ dex at $g<17$. This approach is similar to the traditional $U B V$ method (e.g., Carney 1979), which relies on the strong dependence of $U$-band magnitudes on metal abundance. However, the $u$-band photometry in SDSS is limited to $u \approx 22(99 \%$ detection limit). This, and the greatly deteriorating errors in SDSS $u$-band magnitudes near the faint limit, restricts photometric metallicity estimates to stars with $r \lesssim 20.8$, an insufficient depth to fully explore the VOD. Here we overcome the limitations of the $u$ band photometry in SDSS by exploring less metallicity-sensitive, but better-determined, color indices in the gri passbands. A full description of this method and our results can be found in An et al. (2009b). Here we only discuss the highlights.

\section{Photometric Metallicity Estimates}

The set of stellar isochrones in An et al. (2009a) were used to determine photometric metallicity estimates based on color-color relations for main-sequence stars. A photometric metallicity $\left([\mathrm{Fe} / \mathrm{H}]_{\text {phot }}\right)$ was computed for each star by requiring distances from main-sequence fitting to be the same from two different CMDs, with $g-r$ and $g-i$ as color indices and $r$ as a luminosity index. We searched the entire $[\mathrm{Fe} / \mathrm{H}]$ grid from -3.0 to +0.4 to find $[\mathrm{Fe} / \mathrm{H}]$ with a minimum $\chi^{2}$ value, where we defined

$$
\chi^{2}=\frac{\left(\mu_{g-r}-\bar{\mu}\right)^{2}}{\sigma_{\mu_{g-r}}^{2}}+\frac{\left(\mu_{g-i}-\bar{\mu}\right)^{2}}{\sigma_{\mu_{g-i}}^{2}},
$$

for each star. Here, $\mu$ and $\sigma_{\mu}$ are the distance modulus and its error on each CMD, respectively. The $\bar{\mu}$ is a weighted average distance modulus from the $(g-r, r)$ and $(g-i, r)$ CMDs. In principle, this method can be used for multiple color indices. However, since only three passbands are considered here, the problem is reduced to the traditional manner of determining metal abundances from a color-color diagram. In some of the cases, a star becomes bluer than the main-sequence turnoff as the metallicity increases, either due to a large photometric error or a younger age than our assumed values in the models. If the minimum $\chi^{2}$ was not found, $[\mathrm{Fe} / \mathrm{H}]_{\text {phot }}$ was estimated by extrapolating the difference in distance modulus toward higher $[\mathrm{Fe} / \mathrm{H}]$ values based on theoretical predictions.

Although the accuracy of an individual photometric metallicity estimate for a star obtained with this technique is rather large ( 0.5 dex or more), when the method is applied to stellar populations, random errors are beaten down by the large numbers of 

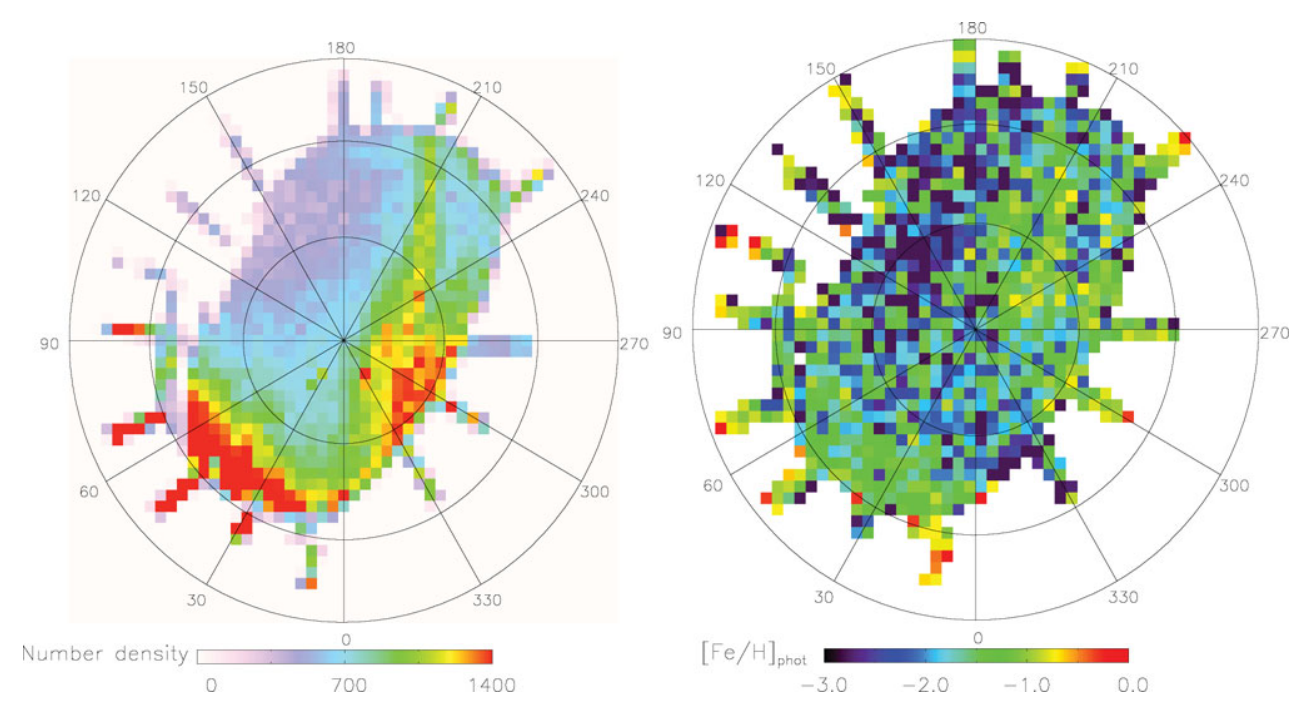

Figure 1. Left: Number density for over 740,000 stars detected in the SDSS, located at distances from $\sim 10 \mathrm{kpc}$ to $\sim 20 \mathrm{kpc}$ from the Sun in the Lambert projection of Galactic coordinates. Each pixel has an area of $12.96 \mathrm{deg}^{2}$, and have a median occupancy of 544 stars per pixel. The North Galactic Pole is at the center, and the Galactic Center is at the bottom. Concentric circles represent $b=0^{\circ}, 30^{\circ}$, and $60^{\circ}$, respectively. The Virgo Overdensity is the feature seen at $(l, b) \sim\left(300^{\circ}, 70^{\circ}\right)$, while the long strip that crosses the sky from $(l, b) \sim\left(210^{\circ}, 30^{\circ}\right)$ to the VOD is the leading tidal tail of Sgr. The feature at $(l, b) \sim\left(40^{\circ}, 40^{\circ}\right)$ is the Hercules-Aquila Cloud, another large-area overdensity of halo stars discovered in SDSS (Belokurov et al. 2007). Right: Median metallicity of the same stars as in the Left panel.

stars involved. In the VOD, for instance (see Figure 1), each pixel in the map is occupied by over 500 stars. The median metallicity of stars in the VOD area at $270^{\circ} \leqslant l \leqslant 330^{\circ}$ and $60^{\circ} \leqslant b \leqslant 70^{\circ}$ is $[\mathrm{Fe} / \mathrm{H}]=-2.0 \pm 0.1$ from the metallicity map in Figure 1 , where the error is from a pixel-to-pixel dispersion.

Field halo stars in the mirrored position of the map shown in Figure 1 at $30^{\circ} \leqslant l \leqslant 90^{\circ}$ and $60^{\circ} \leqslant b \leqslant 70^{\circ}$ exhibit $\langle[\mathrm{Fe} / \mathrm{H}]\rangle=-1.9 \pm 0.1$. Although half of the stars in the direction toward Virgo are likely associated with a progenitor dwarf galaxy or a tidal stream, the mean $[\mathrm{Fe} / \mathrm{H}]$ value essentially remains unchanged; it is the same as that for the field halo stars within the precision of the technique. It is perhaps of interest that the field star metallicities are as low as they appear to be, as Carollo et al. (2007) have argued that the peak of the metallicity of the outer-halo population is $[\mathrm{Fe} / \mathrm{H}]=-2.2$, and that this component is expected to dominate over the more metal-rich inner-halo population (with a peak metallicity at $[\mathrm{Fe} / \mathrm{H}]=-1.6$ ) at Galactocentric distances greater than 15-20 kpc.

\section{A Metallicity Map of the Halo to $20 \mathrm{kpc}$}

The same techniques described above can be used to construct a metallicity map, based on the gri passbands, for main-sequence stars in the SDSS footprint extending well beyond the limit of $9 \mathrm{kpc}$ reached by the metallicity map presented by Ivezić et al. (2008a), based on the ugr passbands. A preliminary result of this exercise is shown in Figure 2. We are still in the process of more fully investigating the effect of photometric errors at low metallicity, however, the current figure certainly suggests that the typical stellar metallicity inferred for stars at larger distances is substantially lower than that for more nearby stars. 


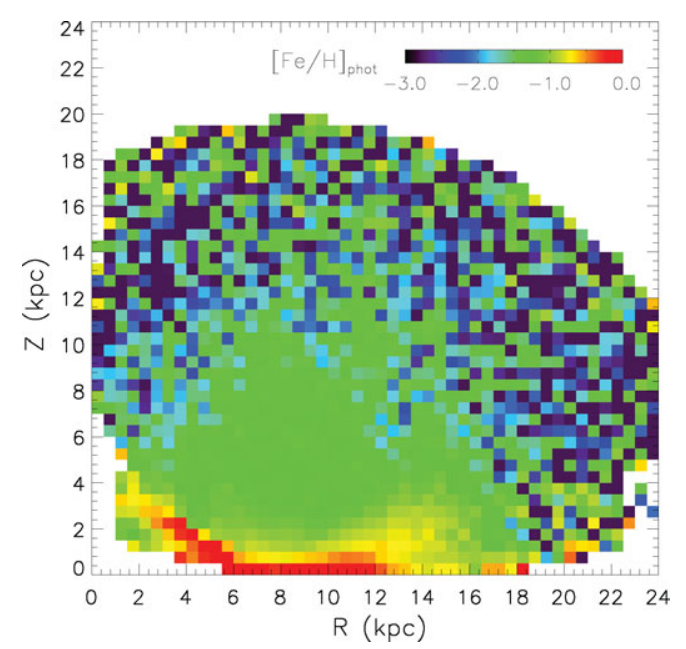

Figure 2. Metallicity map, obtained from photometric metallicity estimates for 2.9 million main-sequence stars with $0.3<g-r<0.4$ in the northern SDSS DR-7 footprint. The median occupancy for pixels shown in the map is 638 stars per pixel. Note that the median metallicity for stars located beyond $10 \mathrm{kpc}$ appears much lower than those within $10 \mathrm{kpc}$.

Carollo et al. (2009) have recently used a new, much larger sample of calibration stars from SDSS DR-7 to develop a more quantitative picture of the inner/outer halo dichotomy. Their derived velocity ellipsoids for these two components - Inner Halo: $\left(\sigma_{V_{R}}\right.$, $\left.\sigma_{V_{\phi}}, \sigma_{V_{Z}}\right)=(150 \pm 2,100 \pm 2,85 \pm 1) \mathrm{km} \mathrm{s}^{-1}$, Outer Halo: $\left(\sigma_{V_{R}}, \sigma_{V_{\phi}}, \sigma_{V_{Z}}\right)=(159 \pm$ $4,165 \pm 9,116 \pm 3) \mathrm{km} \mathrm{s}^{-1}$, clearly demonstrate that outer-halo orbits are significantly less radially elongated than inner-halo orbits, and possess higher energies, consistent with the segregation in spatial dimensions observed in the metallicity map.

Future imaging surveys, such as Pan-STARRS (Kaiser et al. 2002) and LSST (Ivezić et al. 2008b) will use similar photometric bandpasses as those in SDSS, providing even deeper (and far more accurate) photometric data than SDSS over a larger fraction of the sky. Our photometric metallicity method will be useful to exploit these databases for understanding the chemical evolution of progenitor dwarf galaxies that are identified, as well as for the bulk populations of field stars.

\section{References}

An, D. et al. 2009a, ApJ, 700, 523

An, D. et al. 2009b, ApJL, submitted (ArXiv:0907.1082)

Belokurov, V. et al. 2007, ApJ, 657, L89

Bullock, J. S. \& Johnston, K. V. 2005, ApJ, 635, 931

Carney, B. W. 1979, ApJ, 233, 211

Carollo, D. et al. 2007, Nature, 450, 1020

Carollo, D. et al. 2009, ApJ, submitted (ArXiv:0903.3019)

Ivezić, Ž. et al. 2008a, ApJ, 684, 287

Ivezić, Ž. et al. 2008b, ArXiv:0805.2366

Jurić, M. et al. 2008, ApJ, 673, 864

Kaiser, N. et al. 2002, Proc. SPIE, 4836, 154

Newberg, H. J. et al. 2007, ApJ, 668, 221

York, D. G. et al. 2000, AJ, 120, 1579 